\title{
Limnology and Fisheries of the Kushiyara River, Bangladesh
}

\author{
M. M. Islam ${ }^{1}$, S. A. Akhi ${ }^{1}$, M. H. Faruque ${ }^{1,2 *}$ \\ ${ }^{1}$ Department of Fisheries, University of Dhaka, Dhaka-1000, Bangladesh \\ ${ }^{2}$ Department of Environment and Natural Sciences, Yokohama National University, Yokohama, \\ Japan
}

Received 9 April 2018, accepted in final revised form 25 September 2018

\begin{abstract}
A study was conducted in November 2015 to assess the limnological and fisheries conditions of the Kushiyara river, Bangladesh. This study used laboratory techniques, faceto-face interviews and focus group discussions. This study collected water, phytoplankton, zooplankton and benthos from different sites of the river. It found that the water quality parameters such as dissolved oxygen, $\mathrm{pH}$, temperature, turbidity, electrical conductivity, total dissolved solids and sodium chloride of the Kushiyara river are within the standard level for sustaining aquatic life. In the current study, six phytoplankton groups of 19 genera and three groups of zooplankton (4 genera of Rotifers, 3 genera of Copepods and 5 genera of Cladoceras) are recorded. This indicates good ecological conditions of the river water. The current study also recorded 9 groups of benthos, indicating a stable environmental condition in the river for benthos. Diverse kinds of fishing gear were found to operate in this study. They were used to catch almost similar species of fishes using almost similar catching mechanism. The peak and lean seasons for fishing as well as the number, types, length and weight of species of fishes are observed. Overall this study found good water quality and rich biodiversity in the Kushiyara river.
\end{abstract}

Keywords: Water quality; Planktons; Fish population dynamics; Kushiyara river; Bangladesh.

(C) 2019 JSR Publications. ISSN: 2070-0237 (Print); 2070-0245 (Online). All rights reserved. doi: http://dx.doi.org/10.3329/jsr.v11i1.36328 J. Sci. Res. 11 (1), 65-82 (2019)

\section{Introduction}

In a riverine country like Bangladesh, river plays an important role in the country's quest for development. About 800 rivers including tributaries flow through the country constituting a waterway of total length around 24,140 km [1]. Rivers are a great source of freshwater supply and endure multi-species commercial fisheries in our country. Vast amounts of water from rivers are extracted to supply the requirements of urban areas, farms and industries, thus fresh water plays a vital role in human life and economic well-

\footnotetext{
*Corresponding author: faruque-hasan-ny@ynu.jp
} 
being of the society. Production of fishes and other foods and marketable goods, habitat for plants and animals, purification of human and industrial waste, recreation flood control and transportation are other services of fresh-water rivers. These water bodies are inhabited by 260 species of fin-fish, 25 species of prawn and 25 species of turtles [2]. In 2016-2017, the contribution of riverine and estuarine catches to the total fish production of Bangladesh was 6.57 percent (271,639 MT) [3]. Additionally, a substantial number of people are reliant on the river to catch and sell fish to maintain their livelihoods.

A balanced aquatic ecosystem provides several roles and functions, benefiting to mankind. An aquatic ecosystem in good condition can carry out diverse functions such as the production of food and organic matter, the availability of non-renewable resources like water and mineral substance. Moreover, it contributes to stabilize the variability of natural processes (climate, natural risks, etc.) and eliminates the transformation of toxins (water self-purification) and the regulation of ecosystem processes (i.e. climate regulation, hydrological regulation, water purification and treatment, regulation of natural risks and disease, erosion and sedimentation, pollution filtration) [4]. Fresh-water rivers ecosystem is encountering decreases in biodiversity due to anthropogenic and natural causes and if this declining trend proceeds at current rates, the day is not so far when the remaining biodiversity in the fresh-water river will vanish. In Bangladesh, a large portion of total freshwater fish species is facing the threat of extinction. Already 64 fish species have been declared threatened to extinct by IUCN Bangladesh [5]. A total of 30 riverine finfish species have been extinct from Bangladesh [6].

Major threats to freshwater biodiversity were recorded under five headings: overexploitation; flow modification; water pollution; destruction or degradation of habitat; and invasion by non-indigenous species [7]. Their combined and interacting influences have resulted in population declines and range reduction of freshwater biodiversity. Throughout the last century, the riverine ecosystem has suffered from intense human intervention resulting in habitat loss and degradation and as a consequence, many species of fish become highly endangered, particularly in rivers where heavy demand is placed on freshwaters.

The Surma, Kushiyara, Kangsha and Someshwari are considered as major rivers in the north-eastern region of Bangladesh [8]. Fish diversity in Shatghari point of the Surma River and the causes of deterioration of water quality have been assessed $[9,10]$. There are some scattered works on water quality and morphodynamics of the Kushiyara river system [11-13], however, studies on limnological aspects and fisheries conditions of the Kushiyara river has never been reported. Therefore, considering the above motioned research gap, the present study designed to measure the water quality parameters, identify and quantify the phytoplankton, zooplankton and benthos and finally to assess the fish population dynamics through studying fishing gear, fishing effort and catch and fish composition at different stations of the Kushiyara river. 


\section{Materials and Methods}

\subsection{Kushiyara river}

The Kushiyara is one of the major rivers in North-Eastern Bangladesh. It is a transboundary river, originates on the India-Bangladesh border as a branch of the Barak river that enters Bangladesh along $24^{\circ} 53^{\prime}$ north latitudes and $92^{\circ} 32^{\prime}$ east longitudes after flowing westward from Milchar in Kachar district (India). It is about 160 kilometres in length, that carries $85 \%$ flow of the Barak river [14,15]. During the rainy season, the Kushiyara can reach a depth of 10 meters [16]. This river crosses over Zakiganj, Golabganj, Fenchuganj, Balaganj, Rajnagar, Maulvi Bazar, Nabiganj areas of Bangladesh.

\subsection{Sampling stations}

Samples were collected from seven stations: Monumukh (station 1), Alipur (station 2), Char Tajpur (station 3), Parkul (station 4), Paharpur (station 5), Padullah (station 6) and, Jamargaon (station 7) in the Kushiara River (Fig. 1).

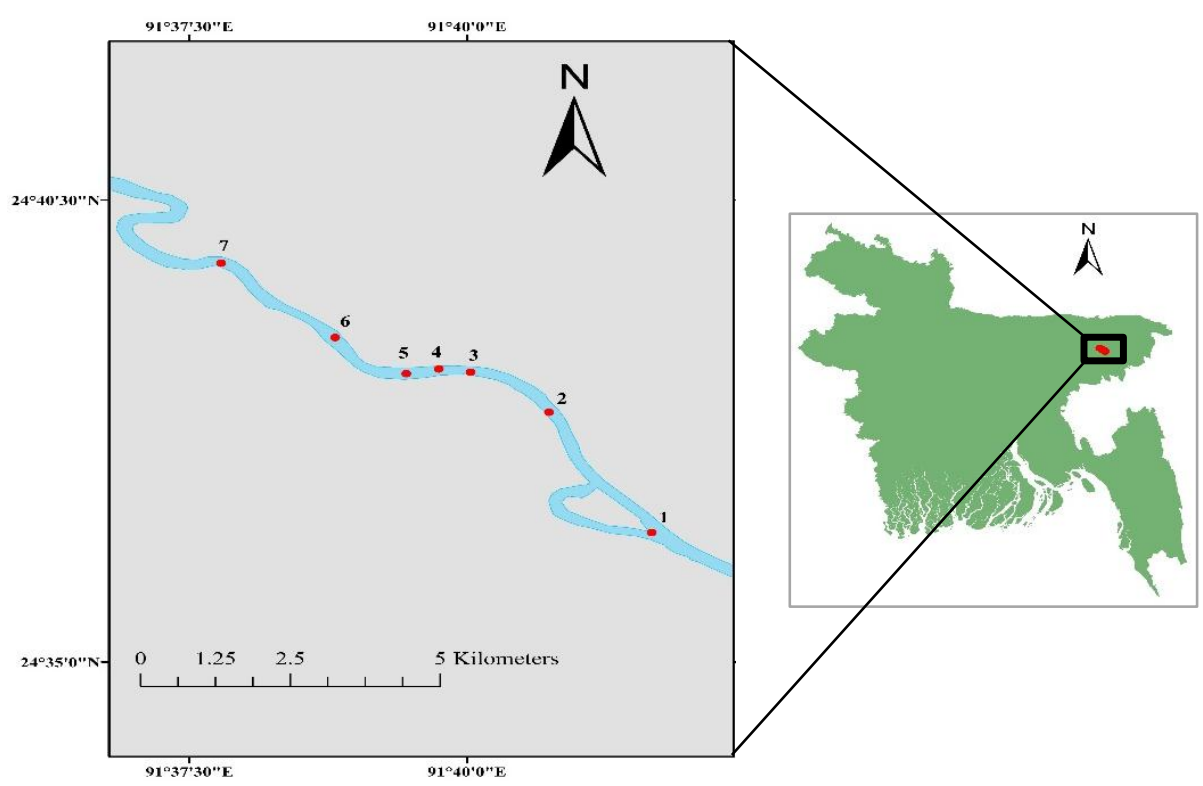

Fig. 1. Locations of the sampling points on the Kushiara River.

\subsection{Limnological survey}

\subsubsection{Water quality parameters}

Water quality parameters such as water temperature, transparency, dissolved oxygen (DO), salinity, pH, electrical conductivity (EC), total dissolved solids (TDS) were 
measured at seven stations in the Kushiyara river. The water temperature and dissolved oxygen were measured on site using $\mathrm{HACH} \mathrm{HQ} 30 \mathrm{~d}$ flexi meter. The turbidity and $\mathrm{pH}$ were measured using Secchi disk transparency and Hanna $\mathrm{pH}$ meter, respectively. The EC, TDS and salinity were measured using Hanna Instruments 2300EC/TDS/ $\mathrm{NaCl}$ meter.

\subsubsection{Phytoplankton and Zooplankton}

Phytoplankton samples were collected from the seven sampling points of the Kushiyara River on $1^{\text {st }}$ November 2015 between 8:30 am to 04:00 pm. Monofilament nylon plankton net of $25 \mu \mathrm{m}$ mesh size (for Phytoplankton) and $50 \mu \mathrm{m}$ mesh size (for Zooplankton) were used for this survey. For the qualitative study, net hauls were made at the surface and at the desired depth $(50 \mathrm{~cm})$ on the river of the sampling sites. For quantitative analysis, both phytoplankton and zooplankton were collected by the plankton net by passing 10 litres of water through it and finally concentrated to $50 \mathrm{~mL}$.

The samples were fixed by addition of $5 \%$ buffered formalin in small plastic bottles to prevent the adverse effects of light and temperature which might cause rapid decay of organisms. Analyses involved the transfer of $1 \mathrm{~mL}$ sub-sample from each of the samples to the Sedgewick-Rafter counter and counting of cells within 10 squares of the cells, chosen randomly under a compound binocular microscope. The cell counts were used to calculate the cell density using the Stirling [17] formula where the plankton density was estimated by-

$N=\frac{A \times 1000 \times C}{V \times F \times L}$

Where, $N$ is no. of phytoplankton or zooplankton cells or units per liter of original river water, $A$ is total no. of phytoplankton or zooplankton counted, $C$ is volume of final concentrate of the samples in $\mathrm{mL}, V$ is volume of a field in cubic mm, $F$ is no. of fields counted, $L$ is volume of original river water in liters.

The phytoplankton and zooplankton were then identified up to the genus level and enumerated with reference to APHA and Bellinger [18,19]. The number of plankton was recorded and expressed numerically per litre of river water. Qualitative studies were undertaken in accordance with APHA, Bellinger, Peenak, Ward and Whipple, Needham and Needham, and Prescott [18-23]. The identification was verified with the specimen at the Department of Fisheries, University of Dhaka.

\subsubsection{Benthos}

Benthos was collected by Ekman dredge at about $3 \mathrm{~m}$ depth (shallow region) from the sampling points of the Kushiyara river. The sample was transferred from the Ekman dredge into a plastic bag on the boat. The sample bags were watertight and transferred to the laboratory in a thermally isolated container cooled with ice packs. The sample was then sieved with sprinkle water directly onto the mud sample with a low-pressure nozzle. The sieving was performed very carefully in order to avoid any damage to the fragile organisms and to ensure that all animal present in the sample was collected. A sieve of 0.5 
$\mathrm{mm}$ mesh was used to isolate macro-fauna. Aquatic vegetation present in the sample was cautiously removed from the surface of the sample, rinsed apart and the resulting water sieved. All material retained on the sieve, including organisms, shell fragments, vegetal debris and coarse sediment grains were transferred to appropriate containers making sure that no other smaller animals were attached to these elements. Containers were labelled indicating the station code, the sample code and sampling date. The sieved material was then fixed to prevent the adverse effects of light and temperature which might cause rapid decay of organisms. Preservation of the samples was done by the addition of $10 \%$ formalin and the samples were analyzed. The volume of the fixative was approximately three times the volume of the sample.

Rose Bengal strain at $200 \mathrm{mg} / \mathrm{L}$ was used carefully to accelerate the sorting procedure. A small quantity of unsorted material was placed on a tray for an initial general sorting for larger organisms with the help of a magnifying lens. Fine sorting was performed under a dissection microscope. During this phase, a small quantity of the sample was spread onto a Petri dish and carefully examined to identify the organisms. Organisms were identified according to the main taxonomic groups, usually bivalves, gastropods, crustaceans, insects etc.

\subsection{Fish population dynamics survey}

A census of all gear operating in the sampling sites was undertaken on daily basis using a gear survey form. Fishing efforts were measured for different gear. Total catch by species was recorded for all gill nets, seine nets, lift net, purse nets, cast nets and long lines operating during that census. Furthermore, the mesh size, owner status and the number of units used by the fishermen were recorded. A catch assessment monitoring questionnaire was developed for the study. Catch monitoring data were collected on a daily basis from the fishermen during fishing. Length-weight frequency data were obtained for key fish species from the fishing boat and two main fish landing centres. To monitor the catch effort, spawning and migration pattern of fishes, the gear orientation (gear set upward or downward direction) was taken into consideration.

Total daily catches by gear type were estimated from their average catch rates and average number of gear recorded daily using a model equation developed by Graff and Chinh [24].

$$
Y_{d}=\sum_{g} \overline{C P U E_{g}} \cdot \overline{f_{g}}
$$

Where, $Y d$ is total daily catch for gear $(\mathrm{g}), C P U E_{g}$ is daily mean catch per unit effort for gear, $f_{g}$ is mean effort (gear/day).

The catch per unit of effort $(C P U E)$ was calculated as the total daily catch $(Y g)$ divided by the sampled gear area and hour fished. 


\subsection{Focus Group Discussion (FGD) and face-to-face interview}

To gather information on fishing gear, fish composition and fish catch, seven FGDs and twenty-one face-to-face interviews were conducted. Each FGD was run for 1.5 to 2 hours and a group of 7 to 8 people from each station were participated. Face-to-face interviews were conducted through a prepared checklist of the questionnaire.

\section{Results}

\subsection{Limnological survey}

\subsubsection{Water quality parameters}

Various physicochemical parameters of the waters of different sampling points are shown in Table 1 . The mean temperature ranged between $28.2^{\circ} \mathrm{C}$ to $29.2{ }^{\circ} \mathrm{C}$. Dissolved oxygen concentration ranged from $6.73-7.05 \mathrm{mg} / \mathrm{L}$. Secchi disk transparency varied between 36 and $50 \mathrm{~cm}$. The $\mathrm{pH}$, EC, TDS and $\mathrm{NaCl}$ range 6.40-6.90, 76.70-81.60 $\mu \mathrm{S} / \mathrm{cm}, 38.60-$ $40.90 \mathrm{mg} / \mathrm{L}$, and $0.1-0.2 \%$, respectively.

Table 1. Water quality parameters at different points of the Kushiara river (Data are represented as mean value).

\begin{tabular}{llllllll}
\hline Study areas & $\begin{array}{l}\text { Temperature } \\
\left({ }^{\circ} \mathrm{C}\right)\end{array}$ & $\begin{array}{l}\text { DO } \\
(\mathrm{mg} / \mathrm{L})\end{array}$ & $\begin{array}{l}\text { Transparency } \\
(\mathrm{cm})\end{array}$ & $\mathrm{pH}$ & $\begin{array}{l}\mathrm{EC} \\
(\mu \mathrm{S} / \mathrm{cm})\end{array}$ & $\begin{array}{l}\text { TDS } \\
(\mathrm{mg} / \mathrm{L})\end{array}$ & $\begin{array}{l}\mathrm{NaCl} \\
(\%)\end{array}$ \\
\hline Monumukh & 28.8 & 6.99 & 50.0 & 6.4 & 81.6 & 40.4 & 0.2 \\
Alipur & 28.9 & 6.73 & 48.5 & 6.9 & 76.7 & 38.1 & 0.1 \\
Char Tajpu & 29.2 & 7.09 & 48.0 & 6.6 & 78.4 & 38.8 & 0.2 \\
Parkul & 28.2 & 7.01 & 49.5 & 6.8 & 81.0 & 40.9 & 0.2 \\
Paharpur & 28.7 & 6.93 & 46.0 & 6.7 & 77.6 & 38.6 & 0.2 \\
Padullah & 28.5 & 7.05 & 43.5 & 6.7 & 80.0 & 40.4 & 0.2 \\
Jamargaon & 28.5 & 7.05 & 36.0 & 6.9 & 77.8 & 38.9 & 0.2 \\
\hline
\end{tabular}

\subsubsection{Phytoplankton and Zooplankton}

In this study, 19 species of phytoplankton belonging to six taxonomic groups were recorded from the Kushiyara river (Table 2) with Chlorophyta presenting as the dominant group followed by Bacillariophytes. Ulothix, under the Chlorophyta group, was identified as the most dominant genus ranging from 30 unit/L at Jamargaon to 400 unit/L at Alipur, followed by Melosira 150 unit/L at Monumukh, under Bacillariophytes group (Table 3). Findings include Volvox ranging from 30 unit/L at Padullah to 80 unit/L at Parkul under Chlorophyta group. Also, a group is newly added that is Pyrrophyta (Genus Ceratium). 
Table 2. Qualitative study of phytoplankton population in the Kushiyara River.

\begin{tabular}{lllllllll}
\hline Group & Genus & $\mathrm{S}^{1} .1$ & S. 2 & S. 3 & S. 4 & S. 5 & S. 6 & S.7 \\
\hline Chlorophyta & Volvox & $\mathrm{P}^{2}$ & $\mathrm{P}$ & $\mathrm{P}$ & $\mathrm{P}$ & $\mathrm{P}$ & $\mathrm{P}$ & $\mathrm{A}^{3}$ \\
& Ankistrodesmus & $\mathrm{P}$ & $\mathrm{P}$ & $\mathrm{A}$ & $\mathrm{A}$ & $\mathrm{A}$ & $\mathrm{A}$ & $\mathrm{A}$ \\
& Spirogyra & $\mathrm{A}$ & $\mathrm{A}$ & $\mathrm{A}$ & $\mathrm{P}$ & $\mathrm{A}$ & $\mathrm{A}$ & $\mathrm{P}$ \\
& Ulothrix & $\mathrm{P}$ & $\mathrm{P}$ & $\mathrm{P}$ & $\mathrm{P}$ & $\mathrm{P}$ & $\mathrm{P}$ & $\mathrm{P}$ \\
& Microspora & $\mathrm{A}$ & $\mathrm{A}$ & $\mathrm{A}$ & $\mathrm{A}$ & $\mathrm{A}$ & $\mathrm{A}$ & $\mathrm{P}$ \\
Pyrrophyta & Clostrium & $\mathrm{A}$ & $\mathrm{A}$ & $\mathrm{P}$ & $\mathrm{A}$ & $\mathrm{A}$ & $\mathrm{A}$ & $\mathrm{A}$ \\
Euglenophyta & Euglium & $\mathrm{P}$ & $\mathrm{P}$ & $\mathrm{A}$ & $\mathrm{P}$ & $\mathrm{A}$ & $\mathrm{A}$ & $\mathrm{A}$ \\
& Lamanea & $\mathrm{P}$ & $\mathrm{P}$ & $\mathrm{P}$ & $\mathrm{P}$ & $\mathrm{P}$ & $\mathrm{P}$ & $\mathrm{P}$ \\
& Trachellomonas & $\mathrm{P}$ & $\mathrm{A}$ & $\mathrm{A}$ & $\mathrm{A}$ & $\mathrm{A}$ & $\mathrm{A}$ & $\mathrm{A}$ \\
Bacillariophyta & Vavicula & $\mathrm{P}$ & $\mathrm{P}$ & $\mathrm{A}$ & $\mathrm{A}$ & $\mathrm{A}$ & $\mathrm{A}$ & $\mathrm{A}$ \\
& Gyrosigma & $\mathrm{A}$ & $\mathrm{A}$ & $\mathrm{P}$ & $\mathrm{A}$ & $\mathrm{A}$ & $\mathrm{P}$ & $\mathrm{A}$ \\
& Synedra & $\mathrm{A}$ & $\mathrm{P}$ & $\mathrm{A}$ & $\mathrm{A}$ & $\mathrm{A}$ & $\mathrm{A}$ & $\mathrm{A}$ \\
& Cymbella & $\mathrm{A}$ & $\mathrm{A}$ & $\mathrm{A}$ & $\mathrm{A}$ & $\mathrm{A}$ & $\mathrm{P}$ & $\mathrm{A}$ \\
& Melosira & $\mathrm{P}$ & $\mathrm{A}$ & $\mathrm{A}$ & $\mathrm{A}$ & $\mathrm{A}$ & $\mathrm{A}$ & $\mathrm{A}$ \\
Desmid & Tabellaria & $\mathrm{P}$ & $\mathrm{A}$ & $\mathrm{A}$ & $\mathrm{A}$ & $\mathrm{A}$ & $\mathrm{A}$ & $\mathrm{A}$ \\
Cyanophyta & Clostridium & $\mathrm{A}$ & $\mathrm{A}$ & $\mathrm{A}$ & $\mathrm{A}$ & $\mathrm{A}$ & $\mathrm{A}$ & $\mathrm{P}$ \\
& Gleocapsa & $\mathrm{A}$ & $\mathrm{P}$ & $\mathrm{A}$ & $\mathrm{A}$ & $\mathrm{A}$ & $\mathrm{A}$ & $\mathrm{A}$ \\
& Oscillatoria & $\mathrm{P}$ & $\mathrm{A}$ & $\mathrm{A}$ & $\mathrm{A}$ & $\mathrm{A}$ & $\mathrm{A}$ & $\mathrm{A}$ \\
\hline
\end{tabular}

${ }^{\text {T }}$ Station, ${ }^{2}$ Present, ${ }^{3}$ Absent

Table 3. Phytoplankton population (unit/L) in the Kushiyara river.

\begin{tabular}{lllllllll}
\hline Group & Genus & S. 1 & S. 2 & S. 3 & S. 4 & S. 5 & S. 6 & S.7 \\
\hline Chlorophyta & Volvox & 50 & 60 & 75 & 80 & 60 & 30 & 0 \\
& Ankistrodesmus & 10 & 10 & 0 & 0 & 0 & 0 & 0 \\
& Spirogyra & 0 & 0 & 0 & 10 & 0 & 0 & 10 \\
& Ulothrix & 110 & 400 & 75 & 250 & 0 & 200 & 30 \\
& Microspora & 0 & 0 & 0 & 0 & 0 & 0 & 10 \\
& Clostrium & 0 & 0 & 20 & 0 & 0 & 0 & 0 \\
Pyrrophyta & Ceratium & 10 & 20 & 0 & 20 & 0 & 0 & 0 \\
Euglenophyta & Euglena & 30 & 10 & 10 & 20 & 30 & 20 & 10 \\
& Lamanea & 20 & 0 & 0 & 0 & 0 & 0 & 0 \\
Bacillariophyta & Trachellomonas & 10 & 0 & 0 & 0 & 0 & 0 & 0 \\
& Navicula & 30 & 50 & 0 & 50 & 20 & 40 & 0 \\
& Gyrosigma & 0 & 0 & 20 & 0 & 0 & 0 & 0 \\
& Synedra & 0 & 10 & 0 & 0 & 0 & 0 & 0 \\
& Cymbella & 0 & 0 & 0 & 0 & 0 & 20 & 0 \\
& Melosira & 150 & 0 & 0 & 0 & 0 & 0 & 0 \\
& Tabellaria & 10 & 0 & 0 & 0 & 0 & 0 & 0 \\
Desmid & Clostridium & 0 & 0 & 0 & 0 & 0 & 0 & 10 \\
Cyanophyta & Gleocapsa & 0 & 100 & 0 & 0 & 0 & 20 & 0 \\
& Oscillatoria & 20 & 0 & 0 & 0 & 0 & 0 & 0 \\
\hline
\end{tabular}

In this study, twelve species of zooplankton belonging to the three taxonomic groups were recorded form the Kushiyara river (Table 4). The most dominant group was Cladocera contributing 5 species, followed by the Rotifera with 4 species and Copedos with 3 species. Moina was found to be the most abundant (21.95\%) genus belonging to 
Cladocera group, ranging from 5 unit/L at Char Tajpur to 15 unit/L at Alipur, whereas at Padullah, Keratella and Branchionus were found in highest number (Table 5).

Table 4. Qualitative study of zooplankton population in Kushiyara river.

\begin{tabular}{lllllllll}
\hline Group & Genus & S. 1 & S. 2 & S. 3 & S. 4 & S. 5 & S. 6 & S.7 \\
\hline Copepods & Diaptomus & P & P & P & P & A & P & P \\
& Cyclops & P & A & P & P & P & A & P \\
& Mesocyclops & A & A & A & P & P & P & A \\
Cladocera & Simocephalus & A & P & P & A & P & A & A \\
& Moina & P & P & P & P & P & P & P \\
& Bosmina & P & P & A & P & P & P & P \\
& Daphnia & P & P & P & P & P & P & P \\
& Diaphanosoma & A & A & A & A & P & A & A \\
Rotifer & Trichocerca & A & A & A & P & A & A & A \\
& Asplanchna & P & A & A & P & P & A & A \\
& Branchionus & A & A & A & P & A & P & P \\
& Keratella & A & P & A & P & A & P & A \\
\hline
\end{tabular}

Table 5. Zooplankton population (unit/L) in Kushiyara river.

\begin{tabular}{lllllllllll}
\hline Group & Genus & S. 1 & S. 2 & S. 3 & S. 4 & S. 5 & S. 6 & S.7 & $\begin{array}{l}\text { Total } \\
\text { Num. }\end{array}$ & \begin{tabular}{l} 
Contibution \\
\hline Copepods
\end{tabular} \\
& Diaptomus & 4 & 5 & 8 & 10 & 0 & 7 & 4 & 38 & 10.30 \\
& Cyclops & 9 & 0 & 4 & 4 & 7 & 0 & 3 & 27 & 7.32 \\
Cladocera & Mesocyclops & 0 & 0 & 0 & 2 & 2 & 3 & 0 & 7 & 1.90 \\
& Simocephalus & 0 & 2 & 4 & 0 & 5 & 0 & 0 & 11 & 2.98 \\
& Moina & 14 & 15 & 5 & 12 & 10 & 12 & 13 & 81 & 21.95 \\
& Bosmina & 8 & 6 & 0 & 4 & 6 & 2 & 3 & 29 & 7.86 \\
& Daphnia & 5 & 8 & 4 & 3 & 7 & 6 & 8 & 41 & 11.11 \\
& Diaphanosoma & 0 & 0 & 0 & 0 & 12 & 0 & 0 & 12 & 3.25 \\
Rotifer & Trichocerca & 0 & 0 & 0 & 8 & 0 & 0 & 0 & 8 & 2.17 \\
& Asplanchna & 3 & 0 & 0 & 2 & 4 & 0 & 0 & 9 & 2.44 \\
& Branchionus & 0 & 0 & 0 & 15 & 0 & 16 & 15 & 46 & 12.47 \\
& Keratella & 0 & 15 & 0 & 20 & 0 & 25 & 0 & 60 & 16.26 \\
\hline
\end{tabular}

\subsubsection{Benthos}

A total of 9 groups of benthos were recorded during the study (Table 6). The dominant group was Pila, found in all sampling sites followed by Melania and Lamellidens. The benthos that found in different sampling sites on the Khushiara river were Chironomus, Lamellidens, Pila, Melania, Planorbis, Aeschna Nymph, Dicranota, Annelid and Crab.

Table 6. Benthos content in the Kushiyara River.

\begin{tabular}{|c|c|c|c|c|c|c|c|c|c|}
\hline $\begin{array}{l}\text { Sampling } \\
\text { point }\end{array}$ & $\begin{array}{l}\text { Chironomus } \\
\text { Larvae }\end{array}$ & Lamellidens & Pila & Melania & Planorbis & $\begin{array}{l}\text { Aeschna } \\
\text { Nymph }\end{array}$ & Dicranota & Annelid & Crab \\
\hline Monumukh & 4 & 4 & 12 & 35 & 5 & 1 & 0 & 2 & 0 \\
\hline Alipur & 0 & 0 & 2 & 0 & 0 & 0 & 0 & 0 & 0 \\
\hline $\begin{array}{l}\text { Char } \\
\text { Tajpur }\end{array}$ & 0 & 0 & 8 & 6 & 0 & 0 & 0 & 0 & 1 \\
\hline
\end{tabular}




\begin{tabular}{|c|c|c|c|c|c|c|c|c|c|}
\hline $\begin{array}{l}\text { Sampling } \\
\text { point }\end{array}$ & $\begin{array}{l}\text { Chironomus } \\
\text { Larvae }\end{array}$ & Lamellidens & Pila & Melania & Planorbis & $\begin{array}{l}\text { Aeschna } \\
\text { Nymph }\end{array}$ & Dicranota & Annelid & Crab \\
\hline Parkul & 5 & 15 & 10 & 0 & 0 & 0 & 2 & 0 & 0 \\
\hline Paharpur & 2 & 5 & 5 & & 10 & 0 & 0 & 0 & 0 \\
\hline Padullah & 0 & 0 & 12 & 6 & 3 & 0 & 0 & 2 & 0 \\
\hline Jamargaon & 0 & 0 & 13 & 4 & 6 & 0 & 0 & 0 & 0 \\
\hline
\end{tabular}

\subsection{Fish population dynamics}

\subsubsection{Fishing gear}

A wide range of fishing gear is used to catch fishes from the Kushiyara river that varies with seasons. During monsoon, fishermen use cast nets and fixed lift nets for catching fish from adjacent floodplains but in winter and summer they mostly use seine net, current net, uthar $^{4}$ net (purse net), hooks, long line etc. Most of the fishermen are engaged in seine netting since it is labour intensive. Seine nets were dominantly used in all study areas followed by uthar net and mobile lift nets, fixed lift net, monofilament gill nets and cast net (Table 7).

A significant number of fishermen and their family members in all survey areas except station 3 and station 5 used to catch fishes by hooks and long line. It was also observed that the number of fixed lift net was more in downstream from the plant site compare to upstream. At station 7, about $80 \%$ fishermen use fixed lift net whereas seine net is mostly operated close to station 4 .

Table 7. Commonly operated fishing gear at different survey point in the Kushiyara river, Bangladesh.

\begin{tabular}{lcccccccc}
\hline Name of the fishing Gear & \multicolumn{7}{c}{ Survey Station } \\
\cline { 2 - 8 } & S.1 & S. 2 & S.3 & S. 4 & S. 5 & S. 6 & S.7 \\
Seine net & $\checkmark$ & $\checkmark$ & $\checkmark$ & $\checkmark$ & $\checkmark$ & $\checkmark$ & $\checkmark$ \\
Fixed Lift net (Bel jal) & $\checkmark$ & $\times$ & $\checkmark$ & $\times$ & $\checkmark$ & $\checkmark$ & $\checkmark$ \\
Mobile Lift net (Dharma jal, Leina jal) & $\checkmark$ & $\checkmark$ & $\checkmark$ & $\times$ & $\checkmark$ & $\checkmark$ & $\times$ \\
Mono-filamentous gill net & $\checkmark$ & $\times$ & $\times$ & $\times$ & $\checkmark$ & $\checkmark$ & $\times$ \\
(Current jal, Phas jal) & & & & & & & \\
Uthar net & $\checkmark$ & $\checkmark$ & $\checkmark$ & $\checkmark$ & $\checkmark$ & $\checkmark$ & $\times$ \\
Cast net (Khepla jal) & $\checkmark$ & $\checkmark$ & $\checkmark$ & $\checkmark$ & $\checkmark$ & $\checkmark$ & $\times$ \\
Hook/Long Line & $\checkmark$ & $\checkmark$ & $\times$ & $\checkmark$ & $\times$ & $\checkmark$ & $\checkmark$ \\
Traps & $\times$ & $\times$ & $\times$ & $\times$ & $\times$ & $\times$ & $\times$ \\
\hline${ }^{4}$ One kind of purse net with the average mesh size of 50-65 mm, is operated for catching large sized fish.
\end{tabular}

Generally, several nets are designed to catch many species while others are used to catch a particular species. There was no selective gear for fishing. Small to large size nets were operated in the study area. Seine nets were largest $\left(3703 \mathrm{~m}^{2}\right)$ in size followed by uthar net $\left(695 \mathrm{~m}^{2}\right)$ (Table 8). Usually, uthar net is used to catch large sized fish (mesh size: $50-65 \mathrm{~mm})$. 
Table 8. Average gear size, mesh size and number of fishermen involved in operating different types of fishing gear in the survey areas.

\begin{tabular}{lllll}
\hline Type of Gear & $\begin{array}{l}\text { Average gear } \\
\text { size }\left(\mathrm{m}^{2}\right)\end{array}$ & $\begin{array}{l}\text { Mesh } \\
(\mathrm{mm})\end{array}$ & $\begin{array}{l}\text { size } \\
\text { Number of fishermen } \\
\text { involved to operate each } \\
\text { net }\end{array}$ \\
\hline Seine net & 3703 & $8-18$ & $10-15$ & \\
Mobile lift net & 103 & 12 & $1-3$ & \\
Fixed lift net & 195 & $12-15$ & $1-2$ & \\
Monofilament gill net & 310 & $20-25$ & 2 & \\
Uthar net & 695 & $50-65$ & 3 & \\
\hline
\end{tabular}

\subsubsection{Fishing effort and catch}

According to the catch data and from face to face interviews with fishermen of survey areas, fish caught was highest for seine net and on an average it was $28.11 \mathrm{~kg} \mathrm{gear}^{-1} \mathrm{day}^{-1}$ followed by fixed lift net $\left(7.26 \mathrm{~kg} \mathrm{gear}^{-1} \mathrm{day}^{-1}\right)$ and mobile lift net $\left(5.46 \mathrm{~kg}_{\text {gear }}{ }^{-1} \mathrm{day}^{-1}\right)$ and the lowest $\left(0.6 \mathrm{~kg} \mathrm{gear}^{-1} \mathrm{day}^{-1}\right)$ by hook (Table 9$)$. Fishermen of the survey areas stated that fish catch reaches its peak during mid-September to mid-November when water starts receding and lowest catch during monsoon (June and July).

Table 9. Daily average catches of fish by fishing location with different gear.

\begin{tabular}{lllllllll}
\hline & \multicolumn{7}{l}{$\begin{array}{l}\text { Station-wise daily average catches } \\
\text { Fishing Gears }\end{array}$} \\
\cline { 2 - 9 } & S. 1 & S. 2 & S. 3 & S. 4 & S. 5 & S. 6 & S.7 & $\begin{array}{l}\text { Mean value of daily } \\
\text { average catch }\end{array}$ \\
\cline { 2 - 10 } Seine net & 32.7 & 30.2 & 26.8 & 22.7 & 29.1 & 28.5 & 26.8 & 28.11 \\
Fixed Lift net & 4.3 & - & 3.6 & - & 4.3 & 8.3 & 15.8 & 7.26 \\
Mobile Lift net & 10.8 & 2.7 & 1.5 & - & 5.8 & 6.5 & - & 5.46 \\
Monofilamentous gill net & 3.8 & - & - & - & 3.1 & 3.2 & - & 3.37 \\
Uthar net (Purse net) & 5.7 & 2.7 & 4.9 & 4.2 & 5.3 & 7.5 & - & 5.1 \\
Cast net & 0.6 & 0.8 & 0.8 & 0.7 & 0.7 & 0.8 & - & 0.73 \\
Hook/Long Line & 0.4 & 0.7 & - & 0.5 & - & 0.8 & - & 0.6 \\
\hline
\end{tabular}

\subsubsection{Fish composition}

A total of 34 species of fishes have been recorded from the study site belonging to 27 genera, 14 families and 7 orders (Table 10). The dominant order was Cypriniformes comprising $35.29 \%$ followed by order Siluriformes, Perciformes, Clupeiformes, Synbranchiforms, Beloniformes and Osteoglossiformes respectively constituting 29.41, $14.71,8.82,5.88,2.94,2.94 \%$ of all the number of species recorded (Fig. 2). The dominant family was Cyprinidae comprising $29 \%$ of the total number of species caught (Fig. 3). Fishermen of the survey areas acknowledged that fish diversity and their abundance are season dependent. Usually, late monsoon (Ashwin-Kartik, mid-September to mid-November) is the major peak season for fishing, whereas monsoon (AsharShravan, mid-June to mid-August) is the lean season for fishing. 


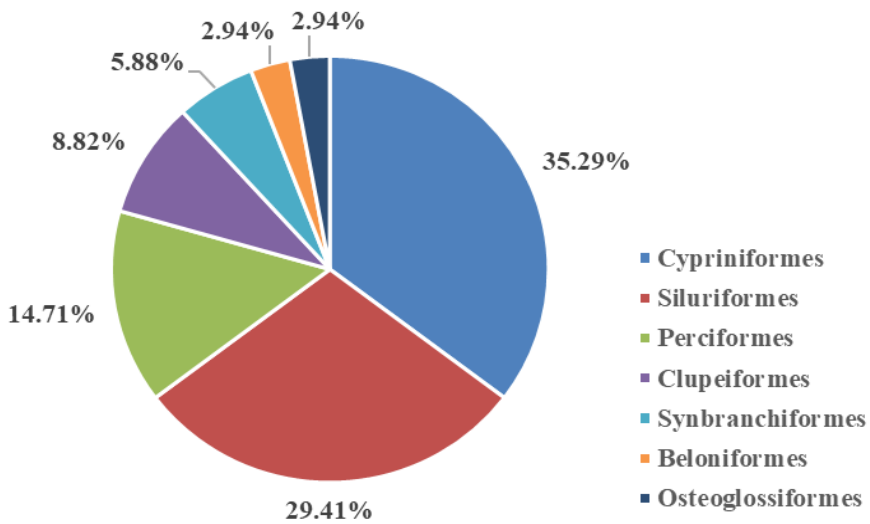

Fig. 2. Order-based fish species diversity in the Kushiyara river.

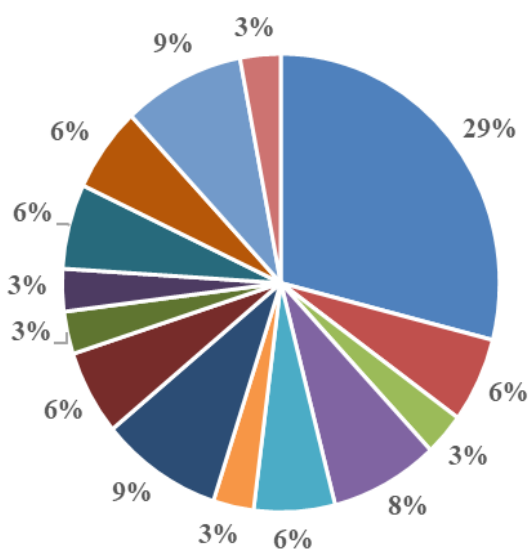

$$
\begin{aligned}
& \text { - Cyprinidae } \\
& \text { - Cobitidae } \\
& \text { - Belonidae } \\
& \text { - Bagridae } \\
& \text { - Siluridae } \\
& \text { - Sisordae } \\
& \text { - Schilbeidae } \\
& \text { - Mastacembelidae } \\
& \text { - Heteropneustidae } \\
& \text { - Notoperidae } \\
& \text { - Ambassidae } \\
& \text { - Channidae } \\
& \text { - Clupeidae } \\
& \text { - Osphronemidae }
\end{aligned}
$$

Fig. 3. Family-based fish species diversity in the survey areas.

According to the FGD respondents, the abundance of fishes by species by Bengali months are shown in the following table:

Table 10. Composition of fish by species (local name) by Bengali months in the river Kushiyara

\begin{tabular}{ll}
\hline Months & Fish species \\
\hline Baisakh & Tenualosa ilisha, Corica soborna, Labeo rohita, Labeo \\
(mid April to mid May) & gonius, Sperata aor, Chanda nama, Labeo calbasu, \\
& Macrobrachium lamarrei, Pangasius bocourti etc. \\
Jaisthya & Tenualosa ilisha, Labeo rohita, Labeo calbasu, \\
(mid May to mid June) & Salmostoma acinaces, Batasio tengana, etc. \\
Ashar & Labeo rohita, Gibelion catla, Labeo calbasu, Cirrhinus \\
\hline
\end{tabular}




\begin{tabular}{|c|c|}
\hline $\operatorname{mid~Ju}$ & $\begin{array}{l}\text { cirrhosus, Ilisha megaloptera, Salmostoma acinaces, } \\
\text { Puntius chola } \text { etc. }\end{array}$ \\
\hline $\begin{array}{l}\text { Shravan } \\
\text { (mid July to mid August) }\end{array}$ & $\begin{array}{l}\text { Labeo rohita, Gibelion catla, Labeo calbasu, Cirrhinus } \\
\text { cirrhosus, Puntius chola, Salmostoma acinaces, Tenualosa } \\
\text { ilisha etc. }\end{array}$ \\
\hline $\begin{array}{l}\text { Vadra } \\
\text { (mid August to mid September) }\end{array}$ & $\begin{array}{l}\text { Wallago attu, Pangasius bocourti, Tenualosa ilisha, } \\
\text { Sperata aor, Amblypharyngodon mola, Chitala chitala, } \\
\text { Labeo gonius, Rita rita, Ilisha megaloptera, etc. }\end{array}$ \\
\hline $\begin{array}{l}\text { Ashwin } \\
\text { (mid September to mid October) }\end{array}$ & $\begin{array}{l}\text { Botia dario, Wallago attu, Sperata aor, Heteropneustes } \\
\text { fossilis, Tenualosa ilisha, Corica soborna, Gibelion catla, } \\
\text { Chitala chitala, Labeo rohita, Labeo gonius, Ailiichthys } \\
\text { punctata, Ilisha megaloptera, Amblypharyngodon mola, } \\
\text { Puntius chola, Chanda nama, Batasio tengana, Labeo } \\
\text { calbasu, Rita rita, Channa striata, Eutropiichthys vacha } \\
\text { etc. }\end{array}$ \\
\hline $\begin{array}{l}\text { Kartik } \\
\text { (mid October to mid November) }\end{array}$ & $\begin{array}{l}\text { Botia dario, Salmostoma acinaces, Rita rita, Chanda nama, } \\
\text { Ailiichthys punctata, Puntius chola, Wallago attu, Anguilla } \\
\text { bengalensis, Batasio tengana, Ilisha megaloptera etc. }\end{array}$ \\
\hline $\begin{array}{l}\text { Agrohayan } \\
\text { (mid November to mid December) }\end{array}$ & $\begin{array}{l}\text { Salmostoma acinaces, Ailiichthys punctata, Chanda nama, } \\
\text { Batasio tengana, Puntius chola, Botia dario, Ilisha } \\
\text { megaloptera etc. }\end{array}$ \\
\hline $\begin{array}{l}\text { Poush } \\
\text { (mid December to mid January) }\end{array}$ & $\begin{array}{l}\text { Batasio tengana, Rita rita, Ilisha megaloptera, Wallago } \\
\text { attu, Pisodonophis cancrivorus etc. }\end{array}$ \\
\hline $\begin{array}{l}\text { Magh } \\
\text { (mid January to mid February }\end{array}$ & $\begin{array}{l}\text { Batasio tengana, Corica soborna, Sperata aor, Tenualosa } \\
\text { ilisha etc. }\end{array}$ \\
\hline to mid March) & $\begin{array}{l}\text { Batasio tengana, Eutropiichthys vacha, Corica soborna, } \\
\text { Tenualosa ilisha, Sperata aor etc. }\end{array}$ \\
\hline $\begin{array}{l}\text { Chaitra } \\
\text { (mid March to mid April) }\end{array}$ & $\begin{array}{l}\text { Tenualosa ilisha, Eutropiichthys vacha, Corica soborna, } \\
\text { Puntius chola, Salmostoma acinaces, Labeo gonius etc. }\end{array}$ \\
\hline
\end{tabular}

According to the survey data, Cirrhinus reba is the most dominant species and it occupied about $50 \%$ of total catch.

Each of the species was assigned the IUCN category of the red list of threatened species for Bangladesh. 10 fish species have been classified threatened out of 34 recorded fish species from the Kushiyara river, which is $29.41 \%$ of the total species assessed. The threatened fishes include 1 species as Critically Endangered (CR), 6 species Endangered (EN) and 3 species as Vulnerable (VU). Total 24 fish species were assessed as Least Concerned (LC) and Not Threatened (NT). No fish was found extinct or regionally extinct.

Table 11. Fish fauna of the river Kushiyara with their status.

\begin{tabular}{lllll}
\hline Order & Family & Scientific name & Local name & Status \\
\hline Cypriniformes & Cyprinidae & Gibelion catla & Katla & LC \\
& & Rabeo rohita & LC \\
& Cirrhinus reba & Lachu & NT \\
& Cirrhinus cirrhosus & Mirka & NT \\
& Labeo calbasu & Kalibaus & LC \\
& Amblypharyngodon mola & Moika & LC \\
& Puntius sophore & Sarputi & LC \\
& Puntius chola & Puti & LC \\
\hline
\end{tabular}




\begin{tabular}{|c|c|c|c|c|}
\hline & & Esomus danricus & Darkina & $\mathrm{LC}$ \\
\hline & & Salmostoma phulo & Chela & NT \\
\hline & Cobitidae & Botia dario & Rani & EN \\
\hline & & Lepidocephalichthys guntea & Gutum & $\mathrm{LC}$ \\
\hline Beloniformes & Belonidae & Xenentodon cancil & Kaika & $\mathrm{LC}$ \\
\hline \multirow[t]{10}{*}{ Siluriformes } & Bagridae & Sperata aor & Ayre & VU \\
\hline & & Mystus tengara & Tangra & $\mathrm{LC}$ \\
\hline & & Rita rita & Rita & EN \\
\hline & Siluridae & Ompok pabda & Pabda & EN \\
\hline & & Wallago attu & Boal & VU \\
\hline & Sisoridae & Bagarius bagarius & Bagair & $\mathrm{CR}$ \\
\hline & Schilbeidae & Ailia coila & Kajoli & $\mathrm{LC}$ \\
\hline & & Clupisoma garua & Gaura & EN \\
\hline & & Eutropiichthys vacha & Bacha & $\mathrm{LC}$ \\
\hline & Heteroneustidae & Heteropneustes fossilis & Shing & $\mathrm{LC}$ \\
\hline \multirow[t]{2}{*}{ Synbranchiformes } & Mastacembelidae & Mastacembelus armatus & Boro Baim & EN \\
\hline & & Macrognathus pancalus & Guchi & $\mathrm{LC}$ \\
\hline Osteoglossiformes & Notopteridae & Chitala chitala & Chital & EN \\
\hline \multirow[t]{5}{*}{ Perciformes } & Ambassidae & Chanda nama & $\begin{array}{l}\text { Nama } \\
\text { chanda }\end{array}$ & $\mathrm{LC}$ \\
\hline & & Parambassis ranga & Lal chanda & $\mathrm{LC}$ \\
\hline & Channidae & Channa striata & Shol & $\mathrm{LC}$ \\
\hline & & Channa punctata & Taki/Cheng & $\mathrm{LC}$ \\
\hline & Osphronemidae & Trichogaster fasciata & Kholisa & $\mathrm{LC}$ \\
\hline \multirow[t]{3}{*}{ Clupeiformes } & Clupeidae & Corica soborna & Keski & $\mathrm{LC}$ \\
\hline & & Gudusia chapra & Chapila & $\mathrm{VU}$ \\
\hline & & Tenualosa ilisha & Ilish & $\mathrm{LC}$ \\
\hline
\end{tabular}

Length-weight relationships for fish were originally used to provide information on the condition of fish and to determine whether somatic growth was isometric or allometric. In this study, the parameters of the length-weight relationships are reported for most available 14 fish species collected from 7 different sampling points and found that there was a strong positive correlation (value ranged from 0.797 to 0.998 ) between length and weight for all sampled species (Table 12 and Fig. 4).

Table 12. Data on length and weight of some fishes of the Kushiyara river (Data are represented as mean \pm standard deviation).

\begin{tabular}{lll}
\hline Name of the fish species & Length $(\mathrm{cm})$ & Weight $(\mathrm{g})$ \\
\hline Puntius chola & $6.7 \pm 0.82$ & $4.6 \pm 1.14$ \\
Channa striata & $34.80 \pm 2.39$ & $337 \pm 46.10$ \\
Botia dario & $7.35 \pm 1.08$ & $3.5 \pm 1.29$ \\
Wallago attu & $50.25 \pm 4.65$ & $574.25 \pm 192.30$ \\
Sperata aor & $24.8 \pm 4.08$ & $72.33 \pm 33.49$ \\
Mystus tengara & $8.22 \pm 1.09$ & $4.17 \pm 1.47$ \\
Trichogaster fasciata & $8.23 \pm 1.24$ & $10 \pm 3.46$ \\
Mastacembelus armatus & $26.66 \pm 1.07$ & $35 \pm 2.55$ \\
Cirrhinus reba & $8.81 \pm 1.18$ & $5.05 \pm 1.12$ \\
Chanda ranga & $5.11 \pm 0.34$ & $1.79 \pm 0.27$ \\
Amblypharyngodon mola & $7.16 \pm 1.24$ & $3.61 \pm 1.34$ \\
Gudusia chapra & $9.51 \pm 1.46$ & $6.75 \pm 3.54$ \\
\hline
\end{tabular}



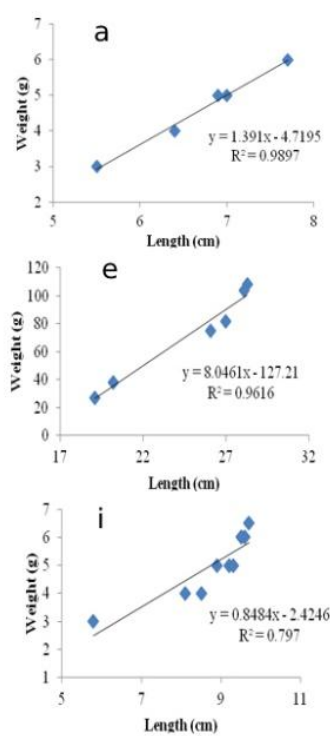
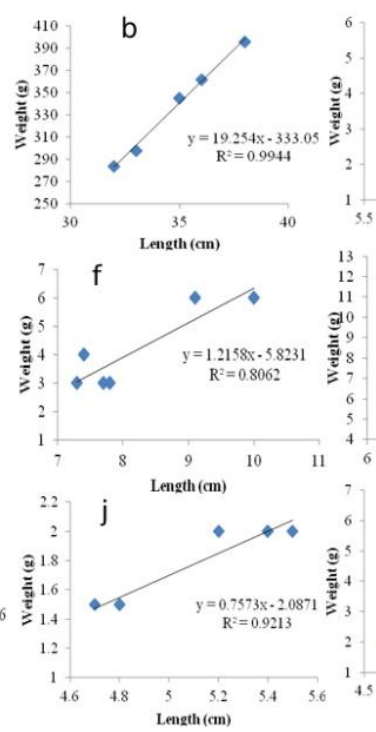

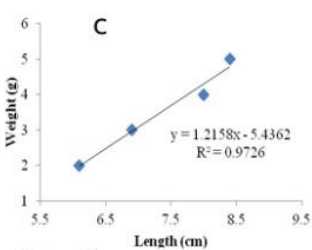

g

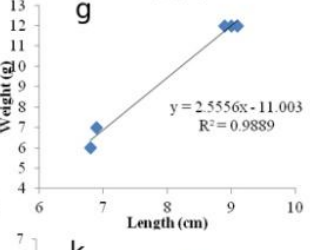

k

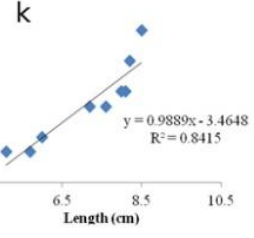

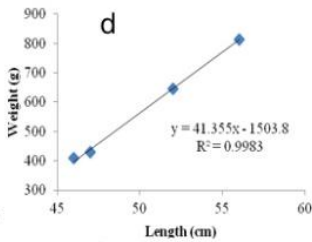

$\mathrm{h}$
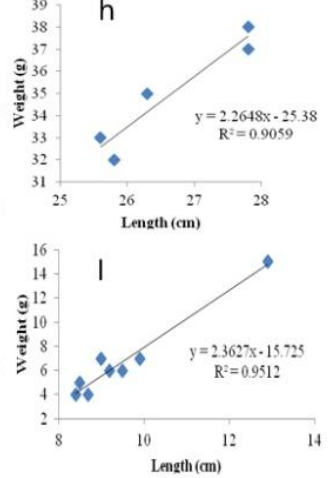

Fig. 4. Length-Weight relationship of fishes found in the Kushiara river: (a) Puntisu chola., (b) Channa striata, (c) Botia dario (d) Wallago attu, (e) Sperata aor, (f) Mystus tengara, (g) Trichogaster fasciata, (h) Mastacembelus armatus, (i) Cirrhinus reba, (j) Chanda ranga, (k) Amblypharyngodon mola and (1) Gudusia chapra.

\section{Discussion}

Information regarding limnological and fisheries conditions at different stations of the Kushiyara river was collected through this present study. The water quality at different stations of the river was measured. Besides, the present status of phytoplankton, zooplankton, and benthos was identified and also the fish population dynamics of different stations of the Kushiyara river were assessed.

In this study, the concentration of $\mathrm{DO}, \mathrm{pH}$, electrical conductivity, total dissolved solids and sodium chloride, all were within the standard range for sustaining aquatic life and Bangladesh national standard for inland surface water (Table 13).

Table 13. Descriptive statistics of the concentration of DO, $\mathrm{pH}, \mathrm{EC}, \mathrm{TDS}, \mathrm{NaCl}$ of the inland surface water at standard level.

\begin{tabular}{lllllll}
\hline Parameters & $\begin{array}{l}\text { Temp. } \\
\left({ }^{\circ} \mathrm{C}\right)\end{array}$ & $\begin{array}{l}\mathrm{DO} \\
(\mathrm{mg} / \mathrm{L})\end{array}$ & $\mathrm{pH}$ & $\begin{array}{l}\mathrm{EC} \\
(\mu \mathrm{S} / \mathrm{cm})\end{array}$ & $\begin{array}{l}\mathrm{TDS} \\
(\mathrm{mg} / \mathrm{L})\end{array}$ & $\begin{array}{l}\mathrm{NaCl} \\
(\%)\end{array}$ \\
\hline $\begin{array}{l}\text { Bangladesh National } \\
\text { Standard for Inland }\end{array}$ & $37.2-46.8$ & $4.5-8$ & $6-9$ & $\leq 1200$ & $\leq 2100$ & \\
$\begin{array}{l}\text { Surface Water } \\
\text { including river }\end{array}$ & & & & & \\
$\begin{array}{l}\text { Standard value for } \\
\text { aquatic life }\end{array}$ & & $4^{[26]}$ & $\begin{array}{l}6.5- \\
9^{[27]}\end{array}$ & & $<500^{[28]}$ & $<0.3^{[27]}$ \\
\hline
\end{tabular}


Dissolved oxygen (DO) is an important ecological variable that decides environmental health of water bodies and support well-balanced aquatic living organisms [29]. In Surma river, the value of DO ranged between 5.52 to $5.72 \mathrm{mg} / \mathrm{L}$ [10] and 4.6 to $5.8 \mathrm{mg} / \mathrm{L}$ in the Meghna river [30] which means the amount of DO in the Kushiyara river ( 6.73 to $7.05 \mathrm{mg} / \mathrm{L}$ ) is higher than the Surma and the Meghna river and it is more suitable for the development of aquatic organism than the two other rivers.

The $\mathrm{pH}$ and electrical conductivity at the different stations of the study area were found within the acceptable range (Table 13) whereas the $\mathrm{pH}$ of the Surma river found slightly acidic and the EC was $84.05 \mu \mathrm{S} / \mathrm{cm}$ [10].

The transparency of productive water bodies should be $40 \mathrm{~cm}$ or less [31]. In the current study, Secchi disk transparency $(36-50 \mathrm{~cm})$ was more than the acceptable limit whereas the transparency level of the Surma river varied from $18.01 \mathrm{~cm}$ (in monsoon) to $48.57 \mathrm{~cm}$ (in winter) with a total mean value of $32.38 \pm 13.46 \mathrm{~cm} \mathrm{[9].}$

Total Dissolved Solids (TDS) are a measure of the amount of particulate solids that are in solution [32]. Average TDS values at the different stations of the study area were found in a very less amount compare to the Karnatoli river (mean value was 152.0 \pm 32.5 $\mathrm{mg} / \mathrm{L})[33]$.

After all, the concentration of physico-chemical parameters at different stations showed that the water quality of the Kushiyara river is sustainable for aquatic life.

The fish bio-diversity in the Kushiyara river showed some similarity with the Surma river. In the Surma river, 34 species of fishes belonging to seven orders were identified [9]. In the Kushiara river, no fish species was found under extinct or regionally extinct. This finding is well coincided with the IUCN's investigation [5].

The phytoplankton diversity is less in the Kushiyara river than the Meghna. In the Meghna, a total of 28 phytoplankton genera were identified in the Meghna river [34]. In the Shitalakhya river, Bacillariophyceae were found to be dominant, which is well coincide with our present finding [35].

Three groups of zooplankton were recorded during the study consisting of 4 genera of Rotifer, 3 genera of Copepods and 5 genera of Cladocera. The most dominant groups were Rotifera and Cladocera whereas, in the Meghna river, Cladocera, Copepod, Rotifer and Sididae were the major groups of Zooplankton [32]. The presence of diverse phytoplankton and zooplankton is indicative of good ecological conditions in the Kushiyara River.

Species diversity of benthos in the study area was relatively low, which may be due to unfavourable condition for organisms in the sampling sites i.e., dragnet fishing was being undertaken in the Kushiyara River near the sampling site, which may adversely affect the total benthos population. However, the abundance of Chironomus larvae and gastropod (Pila, Planorbis, Melania) indicate the good condition of the water body.

In the present investigation, it was found that a wide range of fishing gear is used to catch fishes from the Kushiyara river that varies with seasons and in some case they also used the same fishing net for catching multi species of fish. In this study, the parameters 
of the length-weight relationships show a strong positive correlation (value ranged from 0.797 to 0.998 ) between length and weight for all sampled species.

\section{Conclusion}

The present study was conducted to assess the limnological and fisheries conditions of the Kushiyara river by using modern scientific instruments, laboratory techniques, face-toface interviews and focus group discussions (FGDs). This study did not find any significant natural imbalance in the ecosystem of this river. The water quality of the Kushiyara river is within the standard level for sustaining aquatic life and Bangladesh national standard for inland surface water, good ecological conditions in terms of phytoplankton, zooplankton and benthos populations. However, some factories and power plants which was built on the river bank create noises that may have some negative impacts in future such as damage of fish habitat, increased turbidity, reduced primary productivity, disturbance to fish migration, river bank erosion, damage of fish nursery and spawning grounds, disturbance to fish sanctuary, pollution of the river due to oil spillage from the vessels used for transporting project materials, and disturbance to fish spawning activity. Besides, this study predicts that some of the problems especially degradation of water quality may happen in future due to the same reason as resonated by the FGD participants. They also complained that unplanned dredging activity causing harm to them. In this regards, monitoring should be done at regular intervals (at least once a year) in future to know if there is any negative impact and to take subsequent mitigation and preventive measures.

\section{Acknowledgments}

This research was funded by Summit Power Bangladesh Ltd. Authors are also grateful to Ms. Fatem-Tuz-Zohora, Dept. of Zoology, Jagannath University, M. I. Khan and C. Kar, Dept. of Fisheries, University of Dhaka for their kind cooperation during the period of data collection.

\section{References}

1. CEGIS (Centre for environmental and Geographic Information Services), Ganges River: Morphological Evolution and Prediction (Dhaka, Bangladesh, 2003), pp. 11-13.

2. M. Y. Ali, Fish Resources Vulnerability and Adaptation to Climate Change in Bangladesh. In: Vulnerability and Adaptation to Climate Change for Bangladesh, ed. S. Huq, et al. (Springer, Dordrecht, 1999). https://link.springer.com/chapter/10.1007/978-94-015-9325-0_8

3. DoF, Yearbook of Fisheries Statistics of Bangladesh 2016-17. Fisheries Resources Survey System (FRSS), Department of Fisheries. Bangladesh: Director General, DoF, B 34, 129 (2017).

4. C. Brachet, J. Magnier, D. Valensuela, K. Petit, B. Fribourge-Blanc, N. Bernex, M. Scoullos, and D. Tarlock, The Handbook for Management and Restoration of Aquatic Ecosystems in River and Lake Basins, pp. 94 (2015). 
5. IUCN, Red List of Bangladesh: Freshwater Fishes. International Union for Conservation of Nature, Bangladesh Country Office (Dhaka, Bangladesh, 2015), pp xvi+360.

6. M. A. R. Hossain, Habitat and Fish Diversity: Bangladesh Perspective. pp 1-26 (2014). In: Advances in Fisheries Research in Bangladesh: I - Proc. of 5th Fisheries Conference \& Research Fair, ed. M. A. Wahab et al. (2012).

7. D. Dudgeon, A. H. Arthington, M. O. Gessner, and Z. Kawabata, Biological Rev. 81, 163 (2006). https://doi.org/10.1017/S1464793105006950

8. M. A. R. Hossain, Res. Agric. Livest. Fish. 1, 112 (2014). https://doi.org/10.3329/ralf.v4i3.35093

9. B. Ahammad, M. Khandaker, D. M. I. Hossain, M. A. A. Mamun, S. M. I. Khalil, and S. M. Bari, Int. J. Fish. Aquat. Stud. 5, 205 (2017).

10. J. B. Alam, A. Hossain, S. K. Khan, B. K. Bank, M. R. Islam, Z. Muyen, and M. H. Rahman, Environ. Monit. Assess. 134, 233 (2007). https://www.ncbi.nlm.nih.gov/pubmed/17294273

11. R. Alam and M. D. Hossain, Environ. Monit. Assess. 154, 127 (2009). https://doi.org/10.1007/s10661-008-0383-6

12. M. A. H. Kafi, M. S. Masud, T. K. Magumder, and M. S. Hossain. Assessment of Long Term Evolution of Morphodynamics of the Kalni-Kushiyara River System in Bangladesh using 1Dimensional Morphological Model - Int. Conf. on Recent Innovation in Civil Engineering for Sustainable Development (IICSD-2015) (Gazipur, Bangladesh, 2015).

13. Tajmunnaher, D. M. A. I. Chowdhury, Int. J. New. Technol. Res. 3, 1 (2017).

14. World Boundaries Series, ed. C. Grundy-Warr C (Eurasia) 3, 107 (1994). ISBN 978-0-415-08834-3.

15. "Kushiyara River". Banglapedia: National Encyclopedia of Bangladesh, ed. A. Tahmina et al. (Asiatic Society of Bangladesh, 013).

16. M. Uddin, M. Deb, and D. Das, Remote Sensing Based Analysis of Critical Bends of Kushiyara River in Bangladesh, Geographia Technica. No. 2, 84 (2012).

17. H. P. Stirling, Chemical and Biological methods of Water Analysis for Aquaculturists, (University of Stirling, Scotland. FK94LA, 1985), pp. 119.

18. APHA, Standard Methods for the Examinations of Water and Wastewater, 874 (Washington, DC, New York, 1992).

19. E. G. Bellinger, A Key to Common Algae: Freshwater, Estuarine and Some Coastal Species, $4^{\text {th }}$ Edition (The Institution of Water and Environmental Management, London, 1992), pp.138.

20. R. W. Peenak, Freshwater Invertebrate of the United States (Ronald Press, New York, 1963) pp. 769.

21. H. B. Ward and G. C. Whipple, Freshwater Biology, ed. W. T. Edmondson, $2^{\text {nd }}$ Edition (John Wiley and Sons, New York, 1954) pp. 128

22. J. G. Needham and P. R Needham, A guide to the Study of Freshwater Biology, $5^{\text {th }}$ Edition (Liolden-day, Inc., San Francisco, 1962) pp. 106.

23. G. W. Prescott, Algae of the Western Great Lakes Area (W. C. Brown. Co., Inc. Dubuque. Iowa, 1964), pp. 946. https://doi.org/10.5962/bhl.title.4650

24. G. J. Graaf and N. D. Chinh, Floodplain Fisheries in the Southern Province of Vietnam, (Annual Report, Ministry of Fisheries, 1962) pp. 9.

25. DoE, Guide for Assessment of Effluent Treatment Plants (Department of Environment, Ministry of Environment and Forest, Bangladesh, 2008).

26. M. F. Ahmed and M. M. Rahman, Water Supply and Sanitation: Rural and Low Income Urban Communities, $1^{\text {st }}$ Edition (ITN-Bangladesh, 2000).

27. Environmental Protection Agency (2015). http://www.epa.gov/wqc/national-recommendedwater-quality-criteria-aquatic-life-criteria-table

28. USEPA, Manual of Monitoring Water Quality (1997) 841-B-97-003.

29. B. George, J. I. N. Kumar, and N. R. Kumar, Egypt. J. Aquat. Res. 38, 159 (2012). https://doi.org/10.1016/j.ejar.2016.12.006

30. M. S. Hosaain, N. G. Das, S. Sarker, and M. Z. Rahman, Egypt. J. Aquat. Res. 38, 216 (2012). https://doi.org/10.1016/j.ejar.2016.12.006 
31. M. S. Rahman, Water Quality Management in Aquaculture, 84 (BRAC prokashana, Mohakhali, Dhaka, 1992).

32. Flura, M. A. Alam, A. Nima, M.B. Tanu, and M.H. Khan, Int. J. Fish. Aquat. Stud. 4, 161 (2016).

33. M. K. Ahmed, D. Monika, M. M. Islam, M. S. Akter, S. Islam, and M. A. A. Mansur, World Appl. Sci. J. 12, 155 (2011).

34. A. S. M. Sharif, M. S. Islam, M. N. Hoque, and M. S. Bhuyan, Int. J. Fauna Biol. Stud. 4, 16 (2017).

35. S. M. D. Islam and M. E. Huda, J. Sci. Res. 8, 191 (2016). https://doi.org/10.3329/jsr.v8i2.26402 